\title{
Leading twist asymmetries in deeply virtual Compton scattering.
}

\author{
A.V. Belitsky ${ }^{a}$, D. Müller ${ }^{a, b}$, L. Niedermeier ${ }^{b}$, A. Schäfer $^{b}$ \\ ${ }^{a}$ C.N. Yang Institute for Theoretical Physics \\ State University of New York at Stony Brook \\ NY 11794-3840, Stony Brook, USA \\ ${ }^{b}$ Institut für Theoretische Physik, Universität Regensburg \\ D-93040 Regensburg, Germany
}

\begin{abstract}
We calculate spin, charge, and azimuthal asymmetries in deeply virtual Compton scattering at leading twist-two level. The measurement of these asymmetries gives access to the imaginary and real part of all deeply virtual Compton scattering amplitudes. We note that a consistent description of this process requires taking into account twist-three contributions and we give then a model dependent estimate of these asymmetries.
\end{abstract}

Keywords: deeply virtual Compton scattering, asymmetries, skewed parton distribution PACS numbers: 11.10.Hi, 12.38.Bx, 13.60.Fz 


\section{Introduction.}

The scattering of electroweak probes off hadrons serves as a clean tool, free of complications from hadron-hadron reactions on both the theoretical and experimental sides, for the extraction of reliable information on the substructure of strongly interacting particles. Using the photon as a probe, (the absorptive part of) the forward virtual Compton (VC) process $\gamma^{*}(q) N(P) \rightarrow$ $\gamma^{*}(q) N(P)$ allows to study the strong interaction dynamics and at the same time it has a simple

QCD description in the hard regime, - when $-q^{2} \gg m_{\text {hadr }}^{2}$. In more general settings it is instructive to address the non-forward scattering $\gamma^{(*)}\left(q_{1}\right) N\left(P_{1}\right) \rightarrow \gamma^{(*)}\left(q_{2}\right) N\left(P_{2}\right)$. The systematic approach to its calculation is established only for the deep inelastic domain, where the QCD factorization theorems separate short and long distance phenomena into a perturbative parton subprocess and soft functions which encode information about the strongly coupled regime. The latter, known as the skewed parton distributions (SPDs), being studied for some time [1], have attracted increased attention in light of the conceivable opportunity to learn more about the spin structure of the nucleon [2]. They are also of interest in their own right being hybrids of parton densities/distribution amplitudes and form factors. They share properties of the former in different regions of phase space [3] which have been studied perturbatively to a great extent at one and two-loop orders [4]. Deeply virtual Compton scattering (DVCS), with $q_{2}^{2}=0$, proves to be an experimentally accessible reaction [5]. In electroproduction processes of a real photon there is a strong contamination of DVCS by the Bethe-Heitler $(\mathrm{BH})$ process. In view of the extreme interest to extract, or at least to constrain, SPDs it is timely to address the question of the best observables that allow to get rid of unwanted background. Fortunately, the interference of the two processes provides a rich source of information. It was suggested that diverse asymmetries [6, 2, 7, 8, 9, 10] can be used to disentangle the real and imaginary parts of DVCS and thus give access to SPDs. In the present contribution we consider a number of spin, azimuthal and charge asymmetries which share these properties and give predictions for the kinematics of the HERA and HERMES experiment. Spin asymmetries make it possible to extract the imaginary part of the DVCS amplitude and thus, due to the reality of SPDs, which holds owing to the spatial and time reversal invariance of strong interactions, give directly a measurement of the shape (at leading order in $\alpha_{s}$ in complete analogy to DIS) of SPDs on the diagonal $t=\xi$.

The paper is organized as follows. In section 2 we calculate the squared amplitude for DVCS, $\mathrm{BH}$, and the interference term to leading twist-two accuracy. In section 3 we present simplified formulae for different cross sections and give a qualitative discussion of the feasibility to measure the DVCS leading twist-two amplitudes in different kinematical regions. After introducing models for SPDs, we give an estimate for different asymmetries for HERA and HERMES kinematics in ๒. Finally, in section 5 we summarize. 


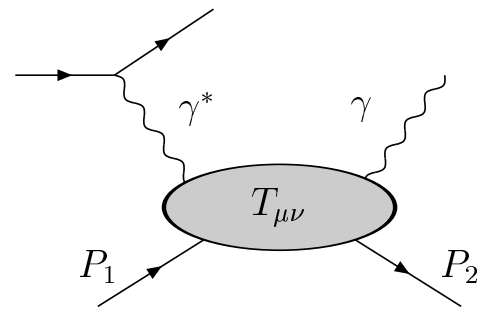

(a)

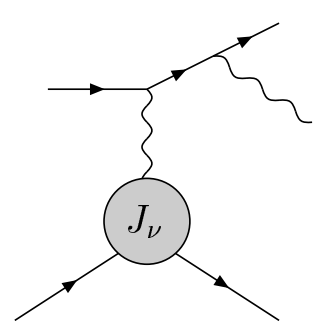

(b)

Figure 1: The virtual Compton scattering amplitude and the Bethe-Heitler process.

\section{Cross section.}

To start let us discuss different contribution to the differential cross section of the electroproduction process $e(k, \lambda) N\left(P_{1}, S_{1}\right) \rightarrow e\left(k^{\prime}, \lambda\right) N\left(P_{2}, S_{2}\right) \gamma\left(q_{2}, \Lambda\right)$, given by the standard formula

$$
d \sigma=\frac{1}{4 k . P_{1}}|\mathcal{T}|^{2}\left(\lambda, S_{1}\right)(2 \pi)^{4} \delta^{4}\left(k+P_{1}-k^{\prime}-P_{2}-q_{2}\right) \frac{d^{3} \boldsymbol{k}^{\prime}}{2 \omega^{\prime}(2 \pi)^{3}} \frac{d^{3} \boldsymbol{P}_{2}}{2 E_{2}(2 \pi)^{3}} \frac{d^{3} \boldsymbol{q}_{2}}{2 \nu(2 \pi)^{3}} .
$$

The scattering amplitude squared $|\mathcal{T}|^{2}$ in the cross section, contains beside the VCS [Fig. П(a)] and $\mathrm{BH}$ [Fig. 目(b) and crossed contribution] parts also the interference term:

$$
|\mathcal{T}|^{2}\left(\lambda, S_{1}\right)=\sum_{\lambda^{\prime}, S_{2}, \Lambda}\left\{\left|\mathcal{T}_{V C S}\right|^{2}+\left|\mathcal{T}_{B H}\right|^{2}+\mathcal{T}_{V C S} \mathcal{T}_{B H}^{*}+\mathcal{T}_{V C S}^{*} \mathcal{T}_{B H}\right\}
$$

The BH-amplitude is purely real and is given as a contraction of the leptonic tensor, at leading order in the fine structure constant $\alpha$,

$$
L_{\mu \nu}=\bar{u}\left(k^{\prime}, \lambda^{\prime}\right)\left[\gamma_{\mu}(\not k-\not \Delta)^{-1} \gamma_{\nu}+\gamma_{\nu}\left(\not k^{\prime}+\not \not\right)^{-1} \gamma_{\mu}\right] u(k, \lambda),
$$

with the hadronic current

$$
J_{\nu}=\bar{U}\left(P_{2}, S_{2}\right)\left\{F_{1}\left(\Delta^{2}\right) \gamma_{\nu}+i F_{2}\left(\Delta^{2}\right) \sigma_{\nu \tau} \frac{\Delta^{\tau}}{2 M}\right\} U\left(P_{1}, S_{1}\right), \quad \text { where } \quad \Delta=P_{2}-P_{1}=q_{1}-q_{2},
$$

parametrized in terms of Dirac, $F_{1}$, and Pauli, $F_{2}$, form factors normalized according to $F_{1}^{p}(0)=1$, $F_{2}^{p}(0) \equiv \kappa_{p}=1.79$, and $F_{1}^{n}(0)=0, F_{2}^{n}(0) \equiv \kappa_{n}=-1.91$, for proton and neutron, respectively. Thus, the $\mathrm{BH}$ amplitude is of the form

$$
\mathcal{T}_{B H}=-\frac{e^{3}}{\Delta^{2}} \epsilon_{\mu}^{*} L^{\mu \nu} J_{\nu}
$$

The form factors are known fairly well from experimental measurements and can be parametrized by dipole formulae in the small $\Delta^{2}$ region

$$
G_{E}^{p}\left(\Delta^{2}\right)=\left(1+\kappa_{p}\right)^{-1} G_{M}^{p}\left(\Delta^{2}\right)=\kappa_{n}^{-1} G_{M}^{n}\left(\Delta^{2}\right)=\left(1-\frac{\Delta^{2}}{m_{V}^{2}}\right)^{-2}, \quad G_{E}^{n}\left(\Delta^{2}\right)=0
$$


where we have introduced the electric, $G_{E}^{i}\left(\Delta^{2}\right)=F_{1}^{i}\left(\Delta^{2}\right)+\frac{\Delta^{2}}{4 M^{2}} F_{2}^{i}\left(\Delta^{2}\right)$, and magnetic, $G_{M}^{i}\left(\Delta^{2}\right)=$ $F_{1}^{i}\left(\Delta^{2}\right)+F_{2}^{i}\left(\Delta^{2}\right)$, form factor characterized by cutoff mass $m_{V}=0.84 \mathrm{GeV}$, see e.g. [13]. The hadronic tensor $T_{V C S}$ is:

$$
\mathcal{T}_{V C S}=\mp \frac{e^{3}}{q_{1}^{2}} \epsilon_{\mu}^{*} T^{\mu \nu} \bar{u}\left(k^{\prime}\right) \gamma_{\nu} u(k), \quad \text { where }\left\{\begin{array}{l}
- \text { for } e^{-} \\
+ \text {for } e^{+}
\end{array} .\right.
$$

It is defined by the time ordered product of two electromagnetic currents

$$
T_{\mu \nu}\left(q, P_{1}, P_{2}\right)=i \int d x e^{i x . q}\left\langle P_{2}, S_{2}\left|T j_{\mu}(x / 2) j_{\nu}(-x / 2)\right| P_{1}, S_{1}\right\rangle,
$$

where $q=\left(q_{1}+q_{2}\right) / 2$ (and the index $\mu$ refers to the outgoing real photon). It contains for a spin-1/2 target twelved independent kinematical structures [6]. In this paper we restrict ourselves to the twist-2 part of $T_{\mu \nu}$ that does not contain transversal photon spin flip contributions. Such contributions arise in the next-to-leading order of perturbation theory due to the gluon transversity [7, 11] and are seperately considered in [12]. From the structure of the OPE we immediately learn that these contributions are contained in the following form factor decomposition']:

$$
T_{\mu \nu}(q, P, \Delta)=-\tilde{g}_{\mu \nu} \frac{q_{\sigma} V_{1}^{\sigma}}{P . q}-i \tilde{\epsilon}_{\mu \nu q \sigma} \frac{A_{1}^{\sigma}}{P . q}+\cdots,
$$

where $P=P_{1}+P_{2}$ and the gauge invariant tensors $\tilde{t}_{\mu \nu}=\mathcal{P}_{\mu \rho} t_{\rho \sigma} \mathcal{P}_{\sigma \nu}$ are constructed by means of the projection tensor $\mathcal{P}_{\mu \nu} \equiv g_{\mu \nu}-q_{1 \mu} q_{2 \nu} / q_{1} . q_{2}$. The ellipsis indicate twist-three and higher contributions. The vectors $V_{i \mu}$ and axial-vectors $A_{i \nu}$ can be expressed by a form factor decomposition

$$
\begin{aligned}
& V_{1 \mu}=\bar{U}\left(P_{2}, S_{2}\right)\left(\mathcal{H}_{1} \gamma_{\mu}+\mathcal{E}_{1} \frac{i \sigma_{\mu \nu} \Delta^{\nu}}{2 M}\right) U\left(P_{1}, S_{1}\right)+\cdots, \\
& A_{1 \mu}=\bar{U}\left(P_{2}, S_{2}\right)\left(\widetilde{\mathcal{H}}_{1} \gamma_{\mu} \gamma_{5}+\widetilde{\mathcal{E}}_{1} \frac{\Delta_{\mu} \gamma_{5}}{2 M}\right) U\left(P_{1}, S_{1}\right)+\cdots,
\end{aligned}
$$

where higher twist contributions are neglected. These form factors depend on the following variables

$$
\xi=\frac{Q^{2}}{P . q}, \quad Q^{2}=-q^{2}=-\frac{1}{4}\left(q_{1}+q_{2}\right)^{2}, \quad \Delta^{2} .
$$

Note that in general (for off-shell final photons) a second scaling variable $\eta=\frac{\Delta . q}{P \cdot q}$ appears, which is related however to $\xi$, i.e. $\eta=-\xi\left(1-\frac{\Delta^{2}}{4 Q^{2}}\right) \approx-\xi$. The amplitudes are given as convolution in $t, \otimes \equiv \int d t$, of perturbatively calculable hard scattering parts with SPDs:

$$
\begin{gathered}
\left\{\begin{array}{c}
\mathcal{H}_{1} \\
\mathcal{E}_{1}
\end{array}\right\}\left(\xi, Q^{2}, \Delta^{2}\right)=T_{1}\left(\xi, Q^{2}, \mu^{2}, t\right) \otimes\left\{\begin{array}{c}
H \\
E
\end{array}\right\}\left(t, \xi, \Delta^{2}, \mu^{2}\right), \\
\left\{\begin{array}{c}
\widetilde{\mathcal{H}}_{1} \\
\widetilde{\mathcal{E}}_{1}
\end{array}\right\}\left(\xi, Q^{2}, \Delta^{2}\right)=\widetilde{T}_{1}\left(\xi, Q^{2}, \mu^{2}, t\right) \otimes\left\{\begin{array}{c}
\widetilde{H} \\
\widetilde{E}
\end{array}\right\}\left(t, \xi, \Delta^{2}, \mu^{2}\right),
\end{gathered}
$$

\footnotetext{
${ }^{1} 12=\frac{1}{2} \times 3$ (virtual photon) $\times 2$ (final photon $) \times 2$ (initial nucleon) $\times 2$ (final nucleon). The reduction factor $1 / 2$ is a result of parity invariance.

${ }^{2}$ We adopt throughout the conventions of [14], e.g. $\epsilon^{0123}=1$
} 


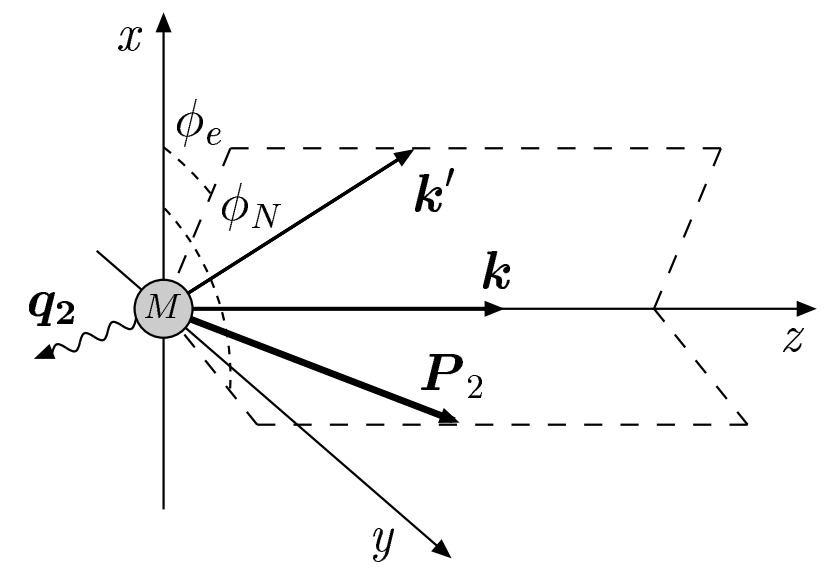

Figure 2: The kinematics of the reaction $e(\boldsymbol{k}) N(M) \rightarrow e\left(\boldsymbol{k}^{\prime}\right) N\left(\boldsymbol{P}_{2}\right) \gamma\left(\boldsymbol{q}_{2}\right)$ in the rest frame of the target.

with summation over the different parton species implied and $\mu^{2}$ being the factorization scale. The hard scattering amplitudes are available in next-to-leading order (NLO) approximation and they read in LO for a quark of charge $Q_{i}$

$$
\xi T^{i(0)}(\xi, t)=\frac{Q_{i}^{2}}{1-t / \xi-i \epsilon} \mp(t \rightarrow-t),
$$

with $-(+)$ for parity even (odd) cases.

In the consequent presentation we give our results in the laboratory frame (see Fig. 目) in which we use the kinematical variables

$$
\begin{aligned}
& k=(E, 0,0, E), \quad k^{\prime}=\left(E^{\prime}, E^{\prime} \cos \phi_{e} \sin \theta_{e}, E^{\prime} \sin \phi_{e} \sin \theta_{e}, E^{\prime} \cos \theta_{e}\right) \\
& P_{1}=(M, 0,0,0), P_{2}=\left(E_{2},\left|\boldsymbol{P}_{2}\right| \cos \phi_{N} \sin \theta_{N},\left|\boldsymbol{P}_{2}\right| \sin \phi_{N} \sin \theta_{N},\left|\boldsymbol{P}_{2}\right| \cos \theta_{N}\right) .
\end{aligned}
$$

Furthermore, we introduce the azimuthal angle $\phi_{r}=\phi_{N}-\phi_{e}$ between the lepton and hadron scattering planes as well as $\phi_{s}=\phi_{N}+\phi_{e}$. The spin vector of the spin- $1 / 2$ target for longitudinal and transverse polarization is given by

$$
S=(0,0,0, \Lambda) \quad \text { with } \quad \Lambda= \pm 1, \quad S=(0, \cos \Phi, \sin \Phi, 0),
$$

respectively.

From the experimental point of view one works with the variables $\mathcal{Q}^{2} \equiv-q_{1}^{2}$ and $x \equiv$ $-q_{1}^{2} /\left(2 P_{1} \cdot q_{1}\right)$, which are related to our variables by

$$
Q^{2}=-\frac{1}{2} q_{1}^{2}\left(1-\frac{\Delta^{2}}{2 q_{1}^{2}}\right) \approx \frac{1}{2} \mathcal{Q}^{2}, \quad \xi=\frac{x\left(1-\frac{\Delta^{2}}{2 q_{1}^{2}}\right)}{2-x\left(1+\frac{\Delta^{2}}{q_{1}^{2}}\right)} \approx \frac{x}{2-x} .
$$

After performing the phase space integration we obtain for the differential cross section (1)

$$
\frac{d \sigma}{d x d \mathcal{Q}^{2} d\left|\Delta^{2}\right| d \phi_{r}}=\frac{y}{\mathcal{Q}^{2}} \frac{d \sigma}{d x d y d\left|\Delta^{2}\right| d \phi_{r}}=\frac{\alpha^{3} x y^{2}}{8 \pi \mathcal{Q}^{4}}\left(1+\frac{4 M^{2} x^{2}}{\mathcal{Q}^{2}}\right)^{-1 / 2}\left|\frac{\mathcal{T}}{e^{3}}\right|^{2},
$$


where we introduced as well the conventional variable $y=P_{1} \cdot q_{1} / P_{1} . k\left[y=1-E^{\prime} / E\right.$ in the frame (15)].

Here we present simple analytical expressions for the amplitudes entering the cross section for positron beam. Changing to electrons will generate a minus sign for the interference terms. To deduce them we present at first the amplitudes squared in terms of the form factors. The result for the DVCS amplitude reads in leading twist-2 approximation (we set $|e|=1$ )

$$
\begin{aligned}
\left|\mathcal{T}_{\mathrm{DVCS}}\right|^{2}= & 8 \frac{2-2 y+y^{2}}{y^{2}} \frac{\xi^{2}}{\mathcal{Q}^{6}}\left(q \cdot V_{1} q \cdot V_{1}^{\dagger}+q \cdot A_{1} q \cdot A_{1}^{\dagger}\right) \\
& +8 \frac{\lambda(2-y)}{y} \frac{\xi^{2}}{\mathcal{Q}^{6}}\left(q \cdot A_{1} q \cdot V_{1}^{\dagger}+q . V_{1} q \cdot A_{1}^{\dagger}\right),
\end{aligned}
$$

where $\lambda=1$ means that the spin of the lepton is parallel to the beam direction. It is obvious that the leading contribution scales as $1 / \mathcal{Q}^{2}$.

The exact squared amplitude for $\mathrm{BH}$ reads in terms of the electromagnetic currents:

$$
\begin{aligned}
\left|\mathcal{T}_{\mathrm{BH}}\right|^{2}= & \frac{8}{\Delta^{2}} \frac{q . J k . J^{\dagger}+k . J q . J^{\dagger}-q . J q . J^{\dagger}-2 k . J k . J^{\dagger}}{\left(2 k . \Delta-\Delta^{2}\right)\left(\mathcal{Q}^{2}+2 k . \Delta\right)} \\
& -\frac{4}{\Delta^{4}}\left(1+\frac{\mathcal{Q}^{4}+\Delta^{4}}{2\left(2 k . \Delta-\Delta^{2}\right)\left(\mathcal{Q}^{2}+2 k . \Delta\right)}\right) J . J^{\dagger} \\
& +\frac{4 i \lambda\left[\left(\mathcal{Q}^{2}+4 k . \Delta-\Delta^{2}\right) \epsilon_{q \Delta J J^{\dagger}}+\Delta^{2} \epsilon_{k(2 q+\Delta) J J^{\dagger}}\right]}{\Delta^{4}\left(2 k . \Delta-\Delta^{2}\right)\left(\mathcal{Q}^{2}+2 k . \Delta\right)},
\end{aligned}
$$

with the latter being defined in Eq. (4). Instead of the exact expression we may use an approximated one. To be consistent we have to expand the squared BH-amplitude, which starts with $1 / \Delta^{2}$, and the interference term up to the same order as the squared DVCS-amplitude, namely to $1 / \mathcal{Q}^{2}$ accuracy. However, one has to take into account that the lepton propagator in the $u$-channel of the $\mathrm{BH}$ amplitude gives a contribution which behaves as $\left(k-q_{2}\right)^{2}=-\frac{(1-y)}{y} \mathcal{Q}^{2}(1+\mathcal{O}(1 / \mathcal{Q}))$. Therefore, the Taylor expansion is not legitimate for large $y$ and sets, otherwise, an upper limit for $y$, namely $1-y \gg M^{2} / \mathcal{Q}^{2}$. To avoid this problem, we have to expand the propagator,

$$
\begin{aligned}
\left(k-q_{2}\right)^{2}= & -\frac{\mathcal{Q}^{2}}{y}\left\{1-y+2 \sqrt{\frac{-\Delta^{2}}{\mathcal{Q}^{2}}} \sqrt{1-\frac{\Delta_{\min }^{2}}{\Delta^{2}}} \sqrt{1-y} \sqrt{1-x} \cos \left(\phi_{r}\right)-\frac{\Delta^{2}}{\mathcal{Q}^{2}}\left[\frac{1-y}{2}\right.\right. \\
& \left.\left.+(1-x)\left(1-2 \frac{\Delta_{\min }^{2}}{\Delta^{2}}\right)-(1-y)\left(x+2(1-x) \frac{\Delta_{\min }^{2}}{\Delta^{2}}\right) \cos \left(2 \phi_{r}\right)\right]+O\left(\frac{1}{\mathcal{Q}^{3}}\right)\right\},
\end{aligned}
$$

and the remaining parts separately in Taylor series which results in a Pade-like approximation for the squared $\mathrm{BH}$ term. Here $\Delta_{\min }^{2}$ is the minimal value of $\Delta^{2}$ which is defined by the kinematical restriction $\Delta_{\min }^{2}=-M^{2} x^{2} /\left(1-x+x M^{2} / \mathcal{Q}^{2}\right)$. Taking only the first terms in the expansion, thus, neglecting all contributions formally suppressed by $1 / \mathcal{Q}$, we get

$$
\left|\mathcal{T}_{\mathrm{BH}}\right|^{2}=-\frac{2\left(2-2 y+y^{2}\right)}{(1-y)}\left(\frac{J . J^{\dagger}}{\Delta^{4}}+4 \frac{q . J q . J^{\dagger}}{\Delta^{2} \mathcal{Q}^{4}}\right)-4 \frac{\lambda(2-y) y^{2}}{(1-y)} \frac{i \epsilon_{k \Delta J J^{\dagger}}}{\Delta^{4} \mathcal{Q}^{2}},
$$


which, however, is valid for $y \ll 1$.

For interference terms we adhere to the same approximation and give here only the leading contributions in $1 / \mathcal{Q}$, i.e.

$$
\begin{aligned}
\mathcal{T}_{\mathrm{BH}} \mathcal{T}_{\mathrm{DVCS}}^{*}= & -4 \frac{2-2 y+y^{2}}{1-y} \frac{\xi}{\Delta^{2} \mathcal{Q}^{4}}\left[\left(k^{\sigma}-\frac{1}{y} q^{\sigma}\right)\left(J_{\sigma}+2 \Delta_{\sigma} \frac{q \cdot J}{\mathcal{Q}^{2}}\right) q \cdot V_{1}^{\dagger}-2 i \epsilon_{k q \Delta J} \frac{q \cdot A_{1}^{\dagger}}{\mathcal{Q}^{2}}\right] \\
& -4 \frac{\lambda(2-y) y}{1-y} \frac{\xi}{\Delta^{2} \mathcal{Q}^{4}}\left[\left(k^{\sigma}-\frac{1}{y} q^{\sigma}\right)\left(J_{\sigma}+2 \Delta_{\sigma} \frac{q \cdot J}{\mathcal{Q}^{2}}\right) q \cdot A_{1}^{\dagger}-2 i \epsilon_{k q \Delta J} \frac{q \cdot V_{1}^{\dagger}}{\mathcal{Q}^{2}}\right] .
\end{aligned}
$$

Note that for a consistent expansion up to order $1 / \mathcal{Q}^{2}$ also twist-three contributions having both kinematical and dynamical origins must be considered (see Ref. [15] for the case of a scalar target). In general these contributions are poorly understood and have to be studied in more detail.

As a next step for the calculation of the cross section we have to sum over the spin of the outgoing proton and write the final answer in terms of SPDs and electromagnetic form factors. Here we give the results for polarized spin- $1 / 2$ target, where $\Lambda=1$ means polarization along the lepton beam, averaged over the azimuthal angle $\phi_{s}$. The DVCS amplitude squared $\left|\mathcal{T}_{\text {DVCS }}\right|^{2}=$ $\left|\mathcal{T}_{\text {DVCS }}\right|_{\text {unp }}^{2}+\left|\mathcal{T}_{\text {DVCS }}\right|_{\text {pol }}^{2}$, with pol $=\{\mathrm{LP}, \mathrm{TP}\}$, consists of the elements

$$
\begin{aligned}
& \left|\mathcal{T}_{\text {DVCS }}\right|_{\text {unp }}^{2}=\frac{2\left(2-2 y+y^{2}\right)}{y^{2}(2-x)^{2} \mathcal{Q}^{2}}\left[4(1-x)\left(\mathcal{H}_{1} \mathcal{H}_{1}^{*}+\widetilde{\mathcal{H}}_{1} \widetilde{\mathcal{H}}_{1}^{*}\right)-x^{2}\left(\mathcal{H}_{1} \mathcal{E}_{1}^{*}+\mathcal{E}_{1} \mathcal{H}_{1}^{*}+\widetilde{\mathcal{H}}_{1} \widetilde{\mathcal{E}}_{1}^{*}+\widetilde{\mathcal{E}}_{1} \widetilde{\mathcal{H}}_{1}^{*}\right)\right. \\
& \left.-\left(x^{2}+(2-x)^{2} \frac{\Delta^{2}}{4 M^{2}}\right) \mathcal{E}_{1} \mathcal{E}_{1}^{*}-x^{2} \frac{\Delta^{2}}{4 M^{2}} \widetilde{\mathcal{E}}_{1} \widetilde{\mathcal{E}}_{1}^{*}\right] \\
& \left|\mathcal{T}_{\mathrm{DVCS}}\right|_{\mathrm{LP}}^{2}=-\frac{2 \lambda \Lambda(2-y)}{y(2-x)^{2} \mathcal{Q}^{2}}\left[4(1-x)\left(\mathcal{H}_{1} \widetilde{\mathcal{H}}_{1}^{*}+\widetilde{\mathcal{H}}_{1} \mathcal{H}_{1}^{*}\right)-x^{2}\left(\mathcal{H}_{1} \widetilde{\mathcal{E}}_{1}^{*}+\widetilde{\mathcal{E}}_{1} \mathcal{H}_{1}^{*}+\widetilde{\mathcal{H}}_{1} \mathcal{E}_{1}^{*}+\mathcal{E}_{1} \widetilde{\mathcal{H}}_{1}^{*}\right)\right. \\
& \left.-x\left(\frac{1}{2} x^{2}+(2-x) \frac{\Delta^{2}}{4 M^{2}}\right)\left(\mathcal{E}_{1} \widetilde{\mathcal{E}}_{1}^{*}+\widetilde{\mathcal{E}}_{1} \mathcal{E}_{1}^{*}\right)\right], \\
& \left|\mathcal{T}_{\mathrm{DVCS}}\right|_{\mathrm{TP}}^{2}=\frac{8 \cos \left(\Phi-\phi_{r} / 2\right)\left(2-2 y+y^{2}\right) \sqrt{1-x}}{\pi y^{2}(2-x)^{2} \mathcal{Q}^{2}} \sqrt{\frac{-\Delta^{2}}{M^{2}}} \sqrt{1-\frac{\Delta_{\min }^{2}}{\Delta^{2}}} \\
& \times\left\{(2-x)\left[\left(\operatorname{Re} \mathcal{H}_{1}\right)\left(\operatorname{Im} \mathcal{E}_{1}\right)-\left(\operatorname{Im} \mathcal{H}_{1}\right)\left(\operatorname{Re} \mathcal{E}_{1}\right)\right]-x\left[\left(\operatorname{Re} \widetilde{\mathcal{H}}_{1}\right)\left(\operatorname{Im} \widetilde{\mathcal{E}}_{1}\right)-\left(\operatorname{Im} \widetilde{\mathcal{H}}_{1}\right)\left(\operatorname{Re} \widetilde{\mathcal{E}}_{1}\right)\right]\right\} \\
& +\frac{2 \lambda \sin \left(\Phi-\phi_{r} / 2\right)(2-y) \sqrt{1-x}}{\pi y(2-x)^{2} \mathcal{Q}^{2}} \sqrt{-\frac{\Delta^{2}}{M^{2}}} \sqrt{1-\frac{\Delta_{\min }^{2}}{\Delta^{2}}}\left[2 x\left(\mathcal{H}_{1} \widetilde{\mathcal{E}}_{1}^{*}+\mathcal{H}_{1}^{*} \widetilde{\mathcal{E}}_{1}\right)\right. \\
& \left.-2(2-x)\left(\widetilde{\mathcal{H}}_{1} \mathcal{E}_{1}^{*}+\widetilde{\mathcal{H}}_{1}^{*} \mathcal{E}_{1}\right)+x^{2}\left(\mathcal{E}_{1} \widetilde{\mathcal{E}}_{1}^{*}+\mathcal{E}_{1}^{*} \widetilde{\mathcal{E}}_{1}\right)\right] .
\end{aligned}
$$

For the BH term we use an analogous decomposition with

$$
\begin{aligned}
\left|\mathcal{T}_{\mathrm{BH}}\right|_{\mathrm{unp}}^{2} & =-\frac{2\left(2-2 y+y^{2}\right)}{(1-y) \Delta^{2}}\left[4 \frac{1-x}{x^{2}}\left(1-\frac{\Delta_{\text {min }}^{2}}{\Delta^{2}}\right) F_{1}^{2}+2\left(F_{1}+F_{2}\right)^{2}+\left(\frac{\Delta^{2}}{\Delta_{\text {min }}^{2}}-1\right) F_{2}^{2}\right], \\
\left|\mathcal{T}_{\mathrm{BH}}\right|_{\mathrm{LP}}^{2} & =\frac{4 \lambda \Lambda(2-y) y}{(1-y) \Delta^{2}}\left(F_{1}+F_{2}\right)\left[2 \frac{1-x}{x}\left(1-\frac{\Delta_{\min }^{2}}{\Delta^{2}}\right) F_{1}+F_{1}+F_{2}\right] \\
\left|\mathcal{T}_{\mathrm{BH}}\right|_{\mathrm{TP}}^{2} & =\frac{8 \lambda \sin \left(\Phi-\phi_{r} / 2\right)(2-y) y \sqrt{1-x}}{\pi(1-y) x M\left(-\Delta^{2}\right)^{3 / 2}} \sqrt{1-\frac{\Delta_{\min }^{2}}{\Delta^{2}}}\left(F_{1}+F_{2}\right)\left(2 x M^{2} F_{1}+\Delta^{2} F_{2}\right)
\end{aligned}
$$


For a polarized lepton beam the interference term is decomposed into a contribution for an unpolarized and an additional one for a polarized target, i.e. $\mathcal{I}^{2} \equiv \mathcal{T}_{\mathrm{BH}} \mathcal{T}_{\mathrm{DVCS}}^{*}+\mathcal{T}_{\mathrm{DVCS}} \mathcal{T}_{\mathrm{BH}}^{*}=$ $\mathcal{I}_{\text {unp }}^{2}(\lambda)+\mathcal{I}_{\text {pol }}^{2}(\lambda)$, where

$$
\begin{aligned}
& \mathcal{I}_{\text {unp }}^{2}(\lambda)=-\frac{8\left(2-2 y+y^{2}\right) \sqrt{1-x}}{\sqrt{1-y} y x \sqrt{-\Delta^{2} \mathcal{Q}^{2}}} \sqrt{1-\frac{\Delta_{\min }^{2}}{\Delta^{2}}} \cos \left(\phi_{r}\right) \operatorname{Re}\left\{F_{1} \mathcal{H}_{1}+\frac{x}{2-x}\left(F_{1}+F_{2}\right) \widetilde{\mathcal{H}}_{1}\right. \\
& \left.-\frac{\Delta^{2}}{4 M^{2}} F_{2} \mathcal{E}_{1}\right\}-\frac{8 \lambda(2-y) \sqrt{1-x}}{\sqrt{1-y} x \sqrt{-\Delta^{2} \mathcal{Q}^{2}}} \sqrt{1-\frac{\Delta_{\min }^{2}}{\Delta^{2}}} \sin \left(\phi_{r}\right) \operatorname{Im}\left\{F_{1} \mathcal{H}_{1}\right. \\
& \left.+\frac{x}{2-x}\left(F_{1}+F_{2}\right) \widetilde{\mathcal{H}_{1}}-\frac{\Delta^{2}}{4 M^{2}} F_{2} \mathcal{E}_{1}\right\} \text {, } \\
& \mathcal{I}_{\mathrm{LP}}^{2}(\lambda)=\frac{8 \Lambda\left(2-2 y+y^{2}\right) \sqrt{1-x}}{\sqrt{1-y} y x \sqrt{-\Delta^{2} \mathcal{Q}^{2}}} \sqrt{1-\frac{\Delta_{\min }^{2}}{\Delta^{2}}} \sin \left(\phi_{r}\right) \operatorname{Im}\left\{\frac{x}{2-x}\left(F_{1}+F_{2}\right)\left(\mathcal{H}_{1}+\frac{x}{2} \mathcal{E}_{1}\right)\right. \\
& \left.+F_{1} \widetilde{\mathcal{H}}_{1}-\frac{x}{2-x}\left(\frac{x}{2} F_{1}+\frac{\Delta^{2}}{4 M^{2}} F_{2}\right) \widetilde{\mathcal{E}}_{1}\right\}+\frac{8 \lambda \Lambda(2-y) \sqrt{1-x}}{\sqrt{1-y} x \sqrt{-\Delta^{2} \mathcal{Q}^{2}}} \sqrt{1-\frac{\Delta_{\min }^{2}}{\Delta^{2}}} \cos \left(\phi_{r}\right) \\
& \times \operatorname{Re}\left\{\frac{x}{2-x}\left(F_{1}+F_{2}\right)\left(\mathcal{H}_{1}+\frac{x}{2} \mathcal{E}_{1}\right)+F_{1} \widetilde{\mathcal{H}}_{1}-\frac{x}{2-x}\left(\frac{x}{2} F_{1}+\frac{\Delta^{2}}{4 M^{2}} F_{2}\right) \widetilde{\mathcal{E}}_{1}\right\}, \\
& \mathcal{I}_{\mathrm{TP}}^{2}(\lambda)=\frac{8\left(2-2 y+y^{2}\right)}{\sqrt{1-y} y x(2-x) \sqrt{\mathcal{Q}^{2} M^{2}}}\left[\frac { \operatorname { c o s } ( \Phi - 3 \phi _ { r } / 2 ) } { 2 \pi } ( 1 - \frac { \Delta _ { \operatorname { m i n } } ^ { 2 } } { \Delta ^ { 2 } } ) ( 1 - x ) \operatorname { I m } \left\{2 F_{2}\left(\mathcal{H}_{1}+\widetilde{\mathcal{H}}_{1}\right)\right.\right. \\
& \left.-\left[(2-x) F_{1}-x F_{2}\right] \mathcal{E}_{1}-x F_{1} \widetilde{\mathcal{E}}_{1}\right\}+\frac{\cos \left(\Phi+\phi_{r} / 2\right)}{2 \pi}\left(\frac{\Delta_{\min }^{2}}{\Delta^{2}}\left(F_{1}+F_{2}\right) \operatorname{Im}\{4(1-x)\right. \\
& \left.\times\left(\mathcal{H}_{1}-\widetilde{\mathcal{H}}_{1}\right)-x^{2}\left(\mathcal{E}_{1}-\widetilde{\mathcal{E}}_{1}\right)\right\}+\left(1-\frac{\Delta_{\text {min }}^{2}}{\Delta^{2}}\right) \operatorname{Im}\left\{2(1-x) F_{2}\left(\mathcal{H}_{1}-\widetilde{\mathcal{H}}_{1}\right)-\left[(2-x) F_{1}\right.\right. \\
& \left.\left.\left.\left.+x F_{2}\right] \mathcal{E}_{1}+x\left(F_{1}+x F_{2}\right) \widetilde{\mathcal{E}}_{1}\right\}\right)\right]+\frac{8 \lambda(2-y)}{\sqrt{1-y}(2-x) x \sqrt{\mathcal{Q}^{2} M^{2}}}\left[\frac{\sin \left(\Phi-3 \phi_{r} / 2\right)}{2 \pi}\right. \\
& \times\left(1-\frac{\Delta_{\text {min }}^{2}}{\Delta^{2}}\right)(1-x) \operatorname{Re}\left\{2 F_{2}\left(\mathcal{H}_{1}+\widetilde{\mathcal{H}}_{1}\right)-\left[(2-x) F_{1}-x F_{2}\right] \mathcal{E}_{1}-x F_{1} \widetilde{\mathcal{E}}_{1}\right\} \\
& -\frac{\sin \left(\Phi+\phi_{r} / 2\right)}{2 \pi}\left(\frac{\Delta_{\min }^{2}}{\Delta^{2}}\left(F_{1}+F_{2}\right) \operatorname{Re}\left\{4(1-x)\left(\mathcal{H}_{1}-\widetilde{\mathcal{H}}_{1}\right)-x^{2}\left(\mathcal{E}_{1}-\widetilde{\mathcal{E}}_{1}\right)\right\}\right. \\
& +\left(1-\frac{\Delta_{\min }^{2}}{\Delta^{2}}\right) \operatorname{Re}\left\{2(1-x) F_{2}\left(\mathcal{H}_{1}-\widetilde{\mathcal{H}}_{1}\right)-\left[(2-x) F_{1}+x F_{2}\right] \mathcal{E}_{1}\right. \\
& \left.\left.\left.+x\left(F_{1}+x F_{2}\right) \widetilde{\mathcal{E}}_{1}\right\}\right)\right] \text {. }
\end{aligned}
$$

\section{Extraction of leading twist-two amplitudes.}

A strong motivation for the measurement of DVCS arises from the fact that the second moment of SPDs in the parity even sector is related to form factors appearing in the decomposition of the symmetric QCD energy-momentum tensor $\Theta_{\mu \nu}$. A gauge-invariant decomposition of the matrix 
element of the angular-momentum tensor, $\mathcal{M}_{\mu \nu, \sigma}=x_{\mu} \Theta_{\sigma \nu}-x_{\nu} \Theta_{\sigma \mu}$, provides, therefore, a separate estimation of the total angular-momentum fraction carried by quarks and gluons [2]. To achieve this goal it is necessary to interpolate the corresponding moments of the SPDs to forward kinematics $\Delta=0$. The new information that is required (and not available from DIS) is contained in the SPDs appearing in the spin-flip amplitude $\mathcal{E}_{1}$. Having the spin puzzle in mind, we give special attention to the problem of extracting $\mathcal{E}_{1}$ from measurements in different kinematical domains.

The simplified explicit expressions (24-32) for the amplitudes squared allow us to discuss the extraction of the leading twist-two amplitudes from future experimental data. Moreover, they allow us to give a qualitative discussion of the ratio for the different cross sections in more detail. Note, however, that the formulae, presented below, are only valid in a small kinematical window and in general not sufficient for numerical estimates. In the following we divide the kinematical region into $\Delta^{2} \approx \Delta_{\min }^{2},\left|\Delta_{\min }^{2}\right|<\left|\Delta^{2}\right|<M^{2}$, and $\left|\Delta_{\min }^{2}\right|<M^{2} \leq\left|\Delta^{2}\right|$. Furthermore, we separately consider the small $x$ region.

\subsection{Small $x$ region.}

Let us start with the small $x$ region, which is for instance relevant for the HERA experiments [5]. We assume that the small $x$ behavior of $\mathcal{E}_{1}$ and $\widetilde{\mathcal{E}}_{1}$ is not one power less than that of $\mathcal{H}_{1}$ and $\widetilde{\mathcal{H}}_{1}$, and, furthermore, that the SPDs in the small $x$ region are essentially determined by the usual parton densities at least in the DGLAP region. It is easy to establish that for small values of $x$ the ratio of the real to imaginary part of the unpolarized amplitude $\mathcal{H}_{1}$ is about 0.3 (by means of dispersion relation the same magnitude has been obtained in Ref. [8]). In the polarized case we find for instance for the Gehrman-Stirling parametrization 21] that this ratio for $\widetilde{\mathcal{H}}_{1}$ is of order $1-1.7$. However, the contribution of the latter is quite small as compared to $\mathcal{H}_{1}$.

Taking into account these numbers, we find that for longitudinally polarized target Eqs. (24,25) can be approximated by:

$$
\begin{aligned}
\left|\mathcal{T}_{\text {DVCS }}\right|^{2} \approx & \frac{2\left(2-2 y+y^{2}\right)}{y^{2} \mathcal{Q}^{2}}\left[\left(\operatorname{Im} \mathcal{H}_{1}\right)^{2}-\frac{\Delta^{2}}{4 M^{2}}\left\{\left(\operatorname{Im} \mathcal{E}_{1}\right)^{2}+\left(\operatorname{Re} \mathcal{E}_{1}\right)^{2}\right\}\right] \\
& -\frac{4 \lambda \Lambda(2-y)}{y \mathcal{Q}^{2}}\left(\operatorname{Im} \mathcal{H}_{1} \operatorname{Im} \widetilde{\mathcal{H}}_{1}+\operatorname{Re} \mathcal{H}_{1} \operatorname{Re} \widetilde{\mathcal{H}}_{1}\right)
\end{aligned}
$$

Contributions containing $\widetilde{\mathcal{E}}_{1}$ are down by two powers of $x$ or they are proportional to $x \frac{\Delta^{2}}{4 M^{2}}$. The transversally polarized part offers an opportunity to measure $\mathcal{E}_{1}$ :

$$
\begin{aligned}
\left|\mathcal{T}_{\mathrm{DVCS}}\right|_{\mathrm{TP}}^{2} \approx & \frac{4 \cos \left(\Phi-\phi_{r} / 2\right)\left(2-2 y+y^{2}\right)}{\pi y^{2} \mathcal{Q}^{2}} \sqrt{\frac{-\Delta^{2}}{M^{2}}} \sqrt{1-\frac{\Delta_{\min }^{2}}{\Delta^{2}}}\left[\left(\operatorname{Re} \mathcal{H}_{1}\right)\left(\operatorname{Im} \mathcal{E}_{1}\right)-\left(\operatorname{Im} \mathcal{H}_{1}\right)\left(\operatorname{Re} \mathcal{E}_{1}\right)\right] \\
& -\frac{4 \lambda \sin \left(\Phi-\phi_{r} / 2\right)(2-y)}{\pi y \mathcal{Q}^{2}} \sqrt{-\frac{\Delta^{2}}{M^{2}}} \sqrt{1-\frac{\Delta_{\min }^{2}}{\Delta^{2}}}\left[\left(\operatorname{Im} \widetilde{\mathcal{H}}_{1}\right)\left(\operatorname{Im} \mathcal{E}_{1}\right)+\left(\operatorname{Re} \widetilde{\mathcal{H}}_{1}\right)\left(\operatorname{Re} \mathcal{E}_{1}\right)\right]
\end{aligned}
$$


The interference term starts with $x^{-1}$, however, since the sea quark and gluonic contributions to the DVCS amplitudes are expected to grow with $x^{-1}$, the DVCS cross section and the interference term have the same $x$ dependence. For the longitudinally polarized case it reads for small $x$ :

$$
\begin{aligned}
\mathcal{I}^{2}= & -\frac{8 \sin \left(\phi_{r}\right) \sqrt{1-\frac{\Delta_{\text {min }}^{2}}{\Delta^{2}}}}{\sqrt{1-y} y x \sqrt{-\Delta^{2} \mathcal{Q}^{2}}} \operatorname{Im}\left\{\lambda(2-y) y\left(F_{1} \mathcal{H}_{1}-\frac{\Delta^{2}}{4 M^{2}} F_{2} \mathcal{E}_{1}\right)-\Lambda\left(2-2 y+y^{2}\right)\right. \\
& \left.\times\left(\frac{x}{2}\left(F_{1}+F_{2}\right) \mathcal{H}_{1}+F_{1} \widetilde{\mathcal{H}}_{1}\right)\right\}-\frac{8 \cos \left(\phi_{r}\right) \sqrt{1-\frac{\Delta_{\min }^{2}}{\Delta^{2}}}}{\sqrt{1-y} y x \sqrt{-\Delta^{2} \mathcal{Q}^{2}}} \operatorname{Re}\left\{\left(2-2 y+y^{2}\right)\right. \\
& \left.\times\left(F_{1} \mathcal{H}_{1}-\frac{\Delta^{2}}{4 M^{2}} F_{2} \mathcal{E}_{1}\right)-\lambda \Lambda(2-y) y\left(\frac{x}{2}\left(F_{1}+F_{2}\right) \mathcal{H}_{1}+F_{1} \widetilde{\mathcal{H}}_{1}\right)\right\} .
\end{aligned}
$$

The interference term might give in future an opportunity to access the imaginary and real part of the linear combination $F_{1} \mathcal{H}_{1}-\frac{\Delta^{2}}{4 M^{2}} F_{2} \mathcal{E}_{1}$ due to the charge and single lepton spin asymmetries for $-\Delta_{\text {min }}^{2}<-\Delta^{2}$. For a polarized proton beam one may extract in an analogous way $\operatorname{Im}\left(x\left(F_{1}+F_{2}\right) \mathcal{H}_{1} / 2+F_{1} \widetilde{\mathcal{H}}_{1}\right)$ and in combination with a polarized lepton beam one also gets the real part of this expression. Note that one has now the opportunity, at least in principle, to extract the DVCS cross section (33) for unpolarized or double spin flip experiments and thus separate $\mathcal{H}_{1}$ from the $\mathcal{E}_{1}$ contributions for the imaginary and real part.

The squared of the $\mathrm{BH}$ term will generally start with $x^{-2}$ (however, the spin dependent part goes only like $x^{-1}$ ) and has therefore a similar $x$ dependence as the other ones, i.e. $x \operatorname{Im} \mathcal{H}_{1} / F_{i}$ is of order one or so, and grows only slightly with increasing $x$. However, in comparison with the squared DVCS term it is multiplied by $y^{2} \mathcal{Q}^{2} / \Delta^{2}$. Thus, one expects that the BH background is not large in the small $y$ and $-\Delta^{2} / \mathcal{Q}^{2}$ region. We conclude from our discussion that the measurement of the unpolarized and longitudinal polarized cross sections as well as single spin asymmetries it is feasible to disentangle the imaginary and real part of $\mathcal{H}_{1}, \widetilde{\mathcal{H}}_{1}$, and $\mathcal{E}_{1}$ at small $x$ and $-\Delta^{2}$ of the order of one $\mathrm{GeV}^{2}$ or larger. For smaller values $\mathcal{E}_{1}$ is in general kinematically reduced. Since $\widetilde{\mathcal{E}_{1}}$ is suppressed at least by one power in $x$, we conclude that this amplitude will not be accessible in the small $x$ region.

As mentioned, if $-\Delta^{2}$ starts to be smaller, the contributions proportional to $\mathcal{E}_{1}$ die out. In the case of its value at the kinematical boundary, i.e. $\Delta^{2} \approx \Delta_{\min }^{2}$, we see that Eqs. (27-29) are quite simple, and, for instance, for a longitudinally polarized target we obtain

$$
\left|\mathcal{T}_{\mathrm{BH}}\right|^{2}=-\frac{4\left(2-2 y+y^{2}\right)}{(1-y) \Delta_{\min }^{2}}\left(F_{1}+F_{2}\right)^{2}+\frac{4 \lambda \Lambda(2-y) y}{(1-y) \Delta_{\min }^{2}}\left(F_{1}+F_{2}\right)^{2} .
$$

Moreover, in this case the interference term for unpolarized or longitudinally polarized target drops out, too. It is worth to have a closer look at the ratio of the DVCS and BH cross section. The unpolarized cross section is essentially governed by the imaginary part of unpolarized parton 
distributions:

$$
\frac{d \sigma_{\mathrm{DVCS}}(\lambda=0, \Lambda=0)}{d \sigma_{\mathrm{BH}}(\lambda=0, \Lambda=0)}=\frac{(1-y) x^{2} M^{2}}{2 y^{2} \mathcal{Q}^{2}} \frac{\operatorname{Im} \mathcal{H}_{1}^{2}}{\left(F_{1}+F_{2}\right)^{2}}
$$

It is remarkable that the ratio of double spin flip (DF) cross sections is proportional to the product of unpolarized and polarized parton distributions:

$$
\frac{d \sigma_{\mathrm{DVCS}}(\lambda=1, \Lambda=1)-d \sigma_{\mathrm{DVCS}}(\lambda=-1, \Lambda=1)}{d \sigma_{\mathrm{BH}}(\lambda=1, \Lambda=1)-d \sigma_{\mathrm{BH}}(\lambda=-1, \Lambda=1)}=\frac{(1-y) x^{2} M^{2}}{y^{2} \mathcal{Q}^{2}} \frac{\operatorname{Im} \mathcal{H}_{1} \operatorname{Im} \widetilde{\mathcal{H}}_{1}+\operatorname{Re} \mathcal{H}_{1} \operatorname{Re} \widetilde{\mathcal{H}}_{1}}{\left(F_{1}+F_{2}\right)^{2}} .
$$

For the sake of completeness we want also to mention that the interference term for a transversally polarized target does not vanish for $\Delta^{2} \approx \Delta_{\min }^{2}$, while both the DVCS and BH cross section are reduced to the unpolarized ones. This interference term is proportional to $\mathcal{H}_{1}-\widetilde{\mathcal{H}}_{1}$ :

$$
\begin{aligned}
\mathcal{I}^{2}(\lambda)= & \frac{16\left(2-2 y+y^{2}\right)}{\sqrt{1-y} y x \sqrt{\mathcal{Q}^{2} M^{2}}} \frac{\cos \left(\Phi+\phi_{r} / 2\right)}{2 \pi}\left(F_{1}+F_{2}\right) \operatorname{Im}\left\{\mathcal{H}_{1}-\widetilde{\mathcal{H}}_{1}\right\} \\
& -\frac{16 \lambda(2-y)}{\sqrt{1-y} x \sqrt{\mathcal{Q}^{2} M^{2}}} \frac{\sin \left(\Phi+\phi_{r} / 2\right)}{2 \pi}\left(F_{1}+F_{2}\right) \operatorname{Re}\left\{\mathcal{H}_{1}-\widetilde{\mathcal{H}}_{1}\right\} .
\end{aligned}
$$

Note that at the kinematical boundary the spin flip contributions are generally suppressed by at least a factor $x^{2}$. Thus, in this kinematical range it is hopeless to extract $\mathcal{E}_{1}$ or $\widetilde{\mathcal{E}}_{1}$ from any experiment.

\subsection{Asymmetries for $\left|\Delta^{2}\right| \gg\left|\Delta_{\min }^{2}\right|$.}

Away from the kinematical boundary $\left|\Delta^{2}\right| \gg\left|\Delta_{\text {min }}^{2}\right|$ we can consider different cross sections to get separately information on the real and imaginary parts of the leading twist-two amplitudes, $\mathcal{H}_{1}, \ldots, \widetilde{\mathcal{E}}_{1}$. Since the BH cross section depends only on the product of the lepton and target polarization, i.e. on $\lambda \Lambda$ or $\lambda f(\Phi)$ with $f(\Phi+\pi)=-f(\Phi)$, we have a direct access to the interference term in polarized experiments to leading order in $1 / \sqrt{\mathcal{Q}^{2}}$. Moreover, the relative sign of the interference term is determined by the charge of the lepton beam. Thus, the following cross sections allow one to get access to the leading twist-two structure functions in the DVCS amplitude. In the approximation used above and neglecting terms $\mathcal{O}\left(\Delta_{\min }^{2} / \Delta^{2}\right)$, they read:

1. Polarized positron beam and unpolarized target:

$$
\begin{aligned}
\Delta_{\mathrm{SL}} d \sigma & \equiv d \sigma^{\uparrow}-d \sigma^{\downarrow} \\
& =-\frac{16(2-y) \sqrt{1-x}}{\sqrt{1-y} x \sqrt{-\Delta^{2} \mathcal{Q}^{2}}} \sin \left(\phi_{r}\right) \operatorname{Im}\left\{F_{1} \mathcal{H}_{1}+\frac{x}{2-x}\left(F_{1}+F_{2}\right) \widetilde{\mathcal{H}}_{1}-\frac{\Delta^{2}}{4 M^{2}} F_{2} \mathcal{E}_{1}\right\} d \mathcal{M}
\end{aligned}
$$


2. Unpolarized positron beam and longitudinally polarized target:

$$
\begin{aligned}
\Delta_{\mathrm{SLN}} d \sigma \equiv & d \sigma_{\uparrow}-d \sigma_{\downarrow}=\frac{16\left(2-2 y+y^{2}\right) \sqrt{1-x}}{\sqrt{1-y} y x \sqrt{-\Delta^{2} \mathcal{Q}^{2}}} \sin \left(\phi_{r}\right) \\
& \times \operatorname{Im}\left\{\frac{x}{2-x}\left(F_{1}+F_{2}\right)\left(\mathcal{H}_{1}+\frac{x}{2} \mathcal{E}_{1}\right)+F_{1} \widetilde{\mathcal{H}}_{1}+\frac{x}{2-x}\left(\frac{x}{2} F_{1}+\frac{\Delta^{2}}{4 M^{2}} F_{2}\right) \widetilde{\mathcal{E}}_{1}\right\} d \mathcal{M}
\end{aligned}
$$

3. Unpolarized positron beam and transversally polarized target: $(\Phi=\{0, \pi\})$ :

$$
\begin{aligned}
\Delta_{\mathrm{STN}} d \sigma \equiv & d \sigma_{\rightarrow}-d \sigma_{\leftarrow}=\frac{16\left(2-2 y+y^{2}\right)}{\sqrt{1-y} y x(2-x) \sqrt{\mathcal{Q}^{2} M^{2}}} \\
\times & {\left[\frac{\cos \left(3 \phi_{r} / 2\right)}{2 \pi}(1-x) \operatorname{Im}\left\{2 F_{2}\left(\mathcal{H}_{1}+\widetilde{\mathcal{H}}_{1}\right)-\left[(2-x) F_{1}-x F_{2}\right] \mathcal{E}_{1}-x F_{1} \widetilde{\mathcal{E}}_{1}\right\}\right.} \\
& +\frac{\cos \left(\phi_{r} / 2\right)}{2 \pi} \operatorname{Im}\left\{2(1-x) F_{2}\left(\mathcal{H}_{1}-\widetilde{\mathcal{H}}_{1}\right)-\left[(2-x) F_{1}+x F_{2}\right] \mathcal{E}_{1}\right. \\
& \left.\left.+x\left(F_{1}+x F_{2}\right) \widetilde{\mathcal{E}}_{1}\right\}\right] d \mathcal{M} .
\end{aligned}
$$

4. Charge asymmetry in unpolarized experiment:

$$
\begin{aligned}
\Delta_{\mathrm{C}}^{\mathrm{unp}} d \sigma \equiv & d^{+} \sigma^{\text {unp }}-d^{-} \sigma^{\text {unp }}=-\frac{16\left(2-2 y+y^{2}\right) \sqrt{1-x}}{\sqrt{1-y} y x \sqrt{-\Delta^{2} \mathcal{Q}^{2}}} \cos \left(\phi_{r}\right) \\
& \times \operatorname{Re}\left\{F_{1} \mathcal{H}_{1}+\frac{x}{2-x}\left(F_{1}+F_{2}\right) \widetilde{\mathcal{H}}_{1}-\frac{\Delta^{2}}{4 M^{2}} F_{2} \mathcal{E}_{1}\right\} d \mathcal{M} .
\end{aligned}
$$

5. Charge asymmetry in double spin-flip experiments with longitudinally polarized target:

$$
\begin{aligned}
\Delta_{\mathrm{C}}^{\mathrm{DFL}} d \sigma \equiv & d^{+} \sigma_{\uparrow}^{\uparrow}-d^{-} \sigma_{\downarrow}^{\downarrow}-\Delta_{\mathrm{C}} d \sigma^{\mathrm{unp}}=\frac{16(2-y) \sqrt{1-x}}{\sqrt{1-y} x \sqrt{-\Delta^{2} \mathcal{Q}^{2}}} \cos \left(\phi_{r}\right) \\
& \times \operatorname{Re}\left\{\frac{x}{2-x}\left(F_{1}+F_{2}\right)\left(\mathcal{H}_{1}+\frac{x}{2} \mathcal{E}_{1}\right)+F_{1} \widetilde{\mathcal{H}}_{1}-\frac{x}{2-x}\left(\frac{x}{2} F_{1}+\frac{\Delta^{2}}{4 M^{2}} F_{2}\right) \widetilde{\mathcal{E}}_{1}\right\} d \mathcal{M}
\end{aligned}
$$

6. Charge asymmetry in double spin-flip experiments with transversally polarized target:

$$
\begin{aligned}
& \Delta_{\mathrm{C}}^{\mathrm{DFT}} d \sigma \equiv d^{+} \sigma_{\rightarrow}^{\uparrow}-d^{-} \sigma_{\leftarrow}^{\downarrow}-\Delta_{\mathrm{C}} d \sigma^{\mathrm{unp}}=-\frac{8(2-y)}{\sqrt{1-y}(2-x) x \sqrt{\mathcal{Q}^{2} M^{2}}} \\
& \times {\left[\frac{\sin \left(3 \phi_{r} / 2\right)}{2 \pi} 2(1-x) \operatorname{Re}\left\{2 F_{2}\left(\mathcal{H}_{1}+\widetilde{\mathcal{H}}_{1}\right)-\left[(2-x) F_{1}-x F_{2}\right] \mathcal{E}_{1}-x F_{1} \widetilde{\mathcal{E}}_{1}\right\}\right.} \\
&+\frac{\sin \left(\phi_{r} / 2\right)}{\pi} \operatorname{Re}\left\{2(1-x) F_{2}\left(\mathcal{H}_{1}-\widetilde{\mathcal{H}}_{1}\right)-\left[(2-x) F_{1}+x F_{2}\right] \mathcal{E}_{1}\right. \\
&\left.\left.+x\left(F_{1}+x F_{2}\right) \widetilde{\mathcal{E}}_{1}\right\}\right] d \mathcal{M},
\end{aligned}
$$


where $d \mathcal{M}=\frac{\alpha^{3} x y}{8 \pi \mathcal{Q}^{2}}\left(1+\frac{4 M^{2} x^{2}}{\mathcal{Q}^{2}}\right)^{-1 / 2} d x d y d\left|\Delta^{2}\right| d \phi_{r}$. Note that for a consequent numerical treatment the $\Delta_{\min }^{2} / \Delta^{2}$ dependence cannot be droped and can be easily restored from Eqs. (30 32).

As we see from this list, the two single spin cross sections of longitudinally polarized beam or target give us information on the imaginary part of two linear combinations of the four leading twist-two amplitudes. The real part of these two quantities is accessible via charge asymmetry in unpolarized or longitudinally double spin flip experiments. Obviously, at low $\left|\Delta^{2}\right|$, i.e. compared to $4 M^{2} \approx 4 \mathrm{GeV}^{2}$, there is a suppression of contributions proportional to $E$ and $\tilde{E}$ SPDs, which are theoretically not well constraint: the contribution proportional to $E$ SPDs completely drops, while $\widetilde{E}$ is suppressed by $x^{2}$. For the kinematics of present experiments $-\Delta^{2} \sim 1 \mathrm{GeV}^{2}$, and thus it is not a good approximation to drop afore mentioned terms, $\Delta^{2} / 4 M^{2} \sim \mathcal{O}(1)$. However, in certain asymmetries these contributions are accompanied by a factor $x$ and thus can be safely discarded at smaller values of $x$. Further constraints separately for the imaginary and real part arise from spin and charge asymmetries for a transversally polarized target. Fortunately, due to different angular dependences, we have indeed two further independent linear combinations of the DVCS amplitudes. But the kinematical pre-factors and an additional angular dependence of the denominator for large $y$, which we have dropped for simplicity, makes their practical use questionable.

Finally, the whole cross section for unpolarized and polarized $\gamma$ production tests the leading twist-two approximation used above. However, to be consistent one has then to expand the $\mathrm{BH}$ and interference terms up to order $1 / \mathcal{Q}^{2}$. As outlined in section 2 this is straightforward for the $\mathrm{BH}$ cross section, while in the interference term new twist-three contributions will enter. These additional terms will be worked out in a forthcoming paper. Here we should only mention that the different azimuthal angle dependence can be used to pick up different combinations of helicity amplitudes, entering at different twist levels. For instance, for small $y$ it is justified to use the derived formulae which imply that all single spin and charge asymmetry cross sections integrated over the azimuthal angle $\phi_{r}$ vanish at twist-two level, for instance,

$$
\int_{0}^{2 \pi} d \phi_{r} \frac{\Delta_{\mathrm{SL}} d \sigma}{d \phi_{r}}=\mathcal{O}\left(1 / \mathcal{Q}^{2}\right) \quad \text { or } \quad \int_{0}^{2 \pi} d \phi_{r} \frac{\Delta_{\mathrm{C}}^{\mathrm{unp}} d \sigma}{d \phi_{r}}=\mathcal{O}\left(1 / \mathcal{Q}^{2}\right)
$$

\section{Numerical estimates.}

To give phenomenological predictions for the asymmetries discussed above we have to specify models for the SPDs. Definitely, this is the main source of uncertainty for the numerical estimates we present in this section. (Of course, the primary goal of experiments is rather to constrain the skewed functions via the theoretical formulae.)

Let us discuss in turn spin non-flip and flip functions. In the former case SPDs have a definite 
limit for forward kinematics when they reduce to the familiar parton densities. For spin non-flip SPDs we choose an oversimplified factorized form of the $\Delta^{2}$ and $(t, \xi)$ dependence of the SPDs:

$$
H^{i}\left(t, \xi, \Delta^{2}, \mathcal{Q}^{2}\right)=F_{1}^{i}\left(\Delta^{2}\right) q^{i}\left(t, \xi, \mathcal{Q}^{2}\right), \quad \widetilde{H}^{i}\left(t, \xi, \Delta^{2}, \mathcal{Q}^{2}\right)=G_{1}^{i}\left(\Delta^{2}\right) \Delta q^{i}\left(t, \xi, \mathcal{Q}^{2}\right),
$$

where $i$ denotes the quark flavour. Here $F_{1}^{i}\left(\Delta^{2}\right)$ and $G^{i}\left(\Delta^{2}\right)$ are elastic parton form factors normalized to unity at the origin, $F_{1}^{i}(0)=G^{i}(0)=1$, and $q\left(t, \xi, \mathcal{Q}^{2}\right)$ as well as $\Delta q\left(t, \xi, \mathcal{Q}^{2}\right)$ are the non-forward functions specified below. The support of these functions is $[-1,1]$, where for $t>0$ we have the quark distribution and for $t<0$ the antiquark distribution, i.e. $\bar{q}\left(t, \xi, \mathcal{Q}^{2}\right)=-q\left(-t, \xi, \mathcal{Q}^{2}\right)$ and $\Delta \bar{q}\left(t, \xi, \mathcal{Q}^{2}\right)=\Delta q\left(-t, \xi, \mathcal{Q}^{2}\right)$. The normalization of $H^{i}\left(t, \xi, \Delta^{2}, \mathcal{Q}^{2}\right)$ and $\widetilde{H}^{i}\left(t, \xi, \Delta^{2}, \mathcal{Q}^{2}\right)$ at $\Delta^{2}=0, \xi=0$ is determined by parton densities $H^{i}(t, 0,0)=q^{i}(t), \widetilde{H}^{i}(t, 0,0)=\Delta q^{i}(t)$. Since the $\xi$ dependence drops out in the first moment we can constrain the $\Delta^{2}$ dependence by sum rules, e.g.

$$
\int_{-1}^{1} d t H^{i}\left(t, \xi, \Delta^{2}\right)=F_{1}^{i}\left(\Delta^{2}\right), \quad \int_{-1}^{1} d t E^{i}\left(t, \xi, \Delta^{2}\right)=F_{2}^{i}\left(\Delta^{2}\right)
$$

with the Dirac and Pauli form factor, respectively. For non-polarized SPDs the valence $u$ and $d$ quark form factors in the proton can be easily deduced from (6) via $F_{I}^{\left(\begin{array}{c}p \\ n\end{array}\right)}=2\left(\begin{array}{c}Q_{u} \\ Q_{d}\end{array}\right) F_{I}^{u}+\left(\begin{array}{c}Q_{d} \\ Q_{u}\end{array}\right) F_{I}^{d}$ which results in

$$
2 F_{I}^{u}\left(\Delta^{2}\right)=2 F_{I}^{p}\left(\Delta^{2}\right)+F_{I}^{n}\left(\Delta^{2}\right), \quad F_{I}^{d}\left(\Delta^{2}\right)=F_{I}^{p}\left(\Delta^{2}\right)+2 F_{I}^{n}\left(\Delta^{2}\right), \quad \text { for } \quad I=1,2 .
$$

For $s$ (or in general, sea) quark contribution in the parity even sector the first moment vanishes and the sum rule (48) does not give any constraint. Nevertheless, the counting rule for elastic form factors tells us that for large $\Delta^{2}$ we have a $\left(\Delta^{2}\right)^{-3}$ behaviour. This suggests the following dipole fit with the mass cutoff $m_{V}$ chosen as for valence quarks:

$$
G_{E}^{\text {sea }}\left(\Delta^{2}\right)=\frac{1}{1+\kappa_{\text {sea }}} G_{M}^{\text {sea }}\left(\Delta^{2}\right)=\left(1-\frac{\Delta^{2}}{m_{V}^{2}}\right)^{-3},
$$

where $\kappa_{\text {sea }}$ will be specified below.

So far the model has been governed by the known forward densities. Unfortunately, a similar reduction is absent for the helicity flip amplitudes. For $E^{i}$ we adopt nevertheless

$$
E^{i}\left(t, \xi, \Delta^{2}\right)=r^{i}(t, \xi) F_{2}^{i}\left(\Delta^{2}\right) \quad \text { with } \quad r^{i}(t, \xi)=q^{i}(t, \xi)
$$

The identification of $r^{i}(t, \xi)$ with $q^{i}(t, \xi)$ ensures the sum rule for valence quark contributions. Note that the parameter $\kappa_{\text {sea }}$ normalizes the sea quark contribution, for instance, $\kappa_{\text {sea }}=0$ provides $E^{\text {sea }}=0$. In the axial vector channel the quark form factors can be read off from the iso-triplet axial form factor $G_{1}^{(3)}\left(\Delta^{2}\right)$ of the $\beta$-decay and related by isotopic symmetry to the form factor in question. The decay constant $g_{A}^{(3)}$ is expressed by Goldberger-Treiman relation $g_{A}^{(3)} \approx \frac{1}{\sqrt{2} M} f_{\pi} g_{\pi N N}$ 
in terms of the pion decay constant $f_{\pi}$, nucleon mass $M$ and $\pi N N$-coupling, $g_{\pi N N}$, and has the numerical value $g_{A}^{(3)}=1.267$ [13]. If we assume the same $\Delta^{2}$-dependence for the iso-singlet $G_{1}^{(0)}\left(\Delta^{2}\right)$ with the same cutoff and a constant $g_{A}^{(0)}$ we get for quarks

$$
G_{1}^{i}\left(\Delta^{2}\right)=\left(1-\frac{\Delta^{2}}{m_{A}^{2}}\right)^{-2}, \quad \text { for } \quad i=\left\{u_{v}, d_{v}\right\}, \quad \text { and } \quad G_{1}^{\text {sea }}\left(\Delta^{2}\right)=\left(1-\frac{\Delta^{2}}{m_{A}^{2}}\right)^{-3}
$$

with the scale $m_{A}=0.9 \mathrm{GeV}$ [13].

Finally for the polarized spin-flip amplitude it was observed [16, 18, 19] that, similar to the $\beta$-decay effective pseudoscalar form factors [13], one can approximate $\widetilde{E}$ at small $\Delta^{2}$ by the pion pole so that one ends up with the model

$$
\widetilde{E}^{u}=\widetilde{E}^{d}=\frac{1}{2 \xi} \theta(\xi-|t|) \phi_{\pi}(t / \xi) g_{\pi}\left(\Delta^{2}\right), \quad \text { with } \quad g_{\pi}\left(\Delta^{2}\right)=\frac{4 g_{A}^{(3)} M^{2}}{m_{\pi}^{2}-\Delta^{2}} \quad \text { and } \quad \phi_{\pi}(x)=\frac{4}{3}\left(1-x^{2}\right) .
$$

It is reliable to assume that the SPDs in the DGLAP region can be modelled by the forward parton distributions measured in inclusive reactions. In the simplest case we assume that this is the only contribution. We refer to this model as the forward parton distribution (FPD) model which has no skewedeness dependence at the input scale $\mathcal{Q}_{0}$ :

$$
(\Delta) q^{i}\left(t, \xi, \mathcal{Q}_{0}^{2}\right)=(\Delta) q^{i}\left(t, \mathcal{Q}_{0}^{2}\right) \quad \text { for } \quad t>0
$$

The contributions for $t<0$ are easily restored by means of symmetry. Although the input distribution does not depend on $\xi$ a small evolution step does generate such a $\xi$-dependence [17]. A further model is based on an proposal for the so-called double distribution (DD) [1], namely,

$$
q\left(t, \xi, Q^{2}\right)=\int_{-1}^{1} d x \int_{-1+|x|}^{1-|x|} d y \delta(x+\xi y-t) f\left(y, x, Q^{2}\right) .
$$

The functional dependence in the $x$-subspace is given by the shape of the forward parton density, $f(x)$, while the $y /[1-|x|]$-dependence of the integrand has to be similar to that of the distribution amplitude. Thus, $f(y, x)$ is given [16] by the product of a forward distribution $f(z)$ (more precisely $q(z)$ for quarks and $z g(z)$ for gluons) with a profile function $\pi$

$$
f(y, x)=\pi(y, x) f(x)
$$

where $\pi$ for quarks and gluons is given by

$$
\pi^{Q}(y, x)=\frac{3}{4} \frac{[1-|x|]^{2}-y^{2}}{[1-|x|]^{3}}, \quad \pi^{G}(y, x)=\frac{15}{16} \frac{\left\{[1-|x|]^{2}-y^{2}\right\}^{2}}{[1-|x|]^{5}} .
$$

Now we are in a position to give numerical estimates for the cross sections defined in the preceding sections. 


\subsection{HERA kinematics.}

In the small $x$ kinematics, $10^{-5} \leq x \leq 10^{-2}$, first estimates for the unpolarized azimuthal and single spin asymmetry has been given in the factorization [8] and BFKL [22 approach. In the following we evaluate numerically different asymmetries in order to demonstrate that $\mathcal{H}_{1}, \widetilde{\mathcal{H}}_{1}$ and $\mathcal{E}_{1}$ are measurable in HERA experiments. In the following we deal with the FPD model, where we equate the SPDs with the parton densities taking the MRS A' [20] and the GS A [21] parametrization at the input scale $\mathcal{Q}^{2}=4 \mathrm{GeV}^{2}$ and using $\bar{u}=\bar{d}=\bar{s} / 2$ as well as $\Delta \bar{u}=\Delta \bar{d}=\Delta \bar{s}$. For simplicity we do not discuss the $\mathcal{Q}^{2}$ dependence of our predictions due to the perturbative evolution.

The unpolarized azimuthal asymmetry is defined by

$$
A=\frac{\int_{-\pi / 2}^{\pi / 2} d \phi_{r} \frac{d \sigma^{\mathrm{unp}}}{d \phi_{r}}-\int_{\pi / 2}^{3 \pi / 2} d \phi_{r} \frac{d \sigma^{\mathrm{unp}}}{d \phi_{r}}}{\int_{0}^{2 \pi} d \phi_{r} \frac{d \sigma^{\mathrm{unp}}}{d \phi_{r}}}
$$

and its first signature has been seen by the ZEUS collaboration [5]. In the approximation (27) the unpolarized squared $\mathrm{BH}$ term is $\phi_{r}$ independent. However, it turned out that this approximation may provide misleading results due to the neglected azimuthal dependence of the $\mathrm{BH}$ process. In Fig. 3(a) it can be seen that the dropped terms cause a strong $\phi_{r}$-dependence and that only for the Pade-type approximation introduced in section 2 [see Eq. (21) for the expansion of the propagator] there is a good agreement with the exact expression. Since the BH cross section is in general suppressed in the upper hemisphere, one may expect quite different predictions depending on the approximations involved. Indeed, for small values of $x$, where $y=\mathcal{Q}^{2} / x s$ with the center of mass energy $s=4 \cdot 27.5 \cdot 820 \mathrm{GeV}^{2}$, we find a strong deviation, even for small $-\Delta^{2}$. Note that the interference term is only taken into account up to order $1 / \sqrt{-\Delta^{2} \mathcal{Q}^{2}}$ and at present it is not clear whether the $1 / \mathcal{Q}^{2}$ term is crucial or not.

To get information on $\left\{F_{1} \mathcal{H}_{1}-\frac{\Delta^{2}}{4 M^{2}} F_{2} \mathcal{E}_{1}\right\}$ in a cleaner way one may use the azimuthal asymmetry of the charge asymmetry $\Delta_{C}^{\text {unp }} d \sigma=d^{-} \sigma^{\text {unp }}-d^{+} \sigma^{\text {unp }}$ defined in Eq. (43) (in the following we restore the $\Delta_{\min }^{2} / \Delta^{2}$ dependence in the interference amplitudes):

$$
A_{\mathrm{C}}=\frac{\int_{-\pi / 2}^{\pi / 2} d \phi_{r} \frac{\Delta_{C}^{\mathrm{unp}} d \sigma}{d \phi_{r}}-\int_{\pi / 2}^{3 \pi / 2} d \phi_{r} \frac{\Delta_{C}^{\mathrm{unp}} d \sigma}{d \phi_{r}}}{\int_{0}^{2 \pi} d \phi_{r} \frac{d^{-} \sigma^{\mathrm{unp}}+d^{+} \sigma^{\mathrm{unp}}}{d \phi_{r}}},
$$

and the single (lepton) spin asymmetry with unpolarized target $\Delta_{\mathrm{SL}}=d \sigma^{\uparrow}-d \sigma^{\downarrow}$ defined in Eq. (40):

$$
A_{\mathrm{SL}}=\frac{\int_{0}^{\pi} d \phi_{r} \frac{\Delta_{\mathrm{SL}} d \sigma}{d \phi_{r}}-\int_{\pi}^{2 \pi} d \phi_{r} \frac{\Delta_{\mathrm{SL}} d \sigma}{d \phi_{r}}}{\int_{0}^{2 \pi} d \phi_{r} \frac{d \sigma^{\uparrow}+d \sigma^{\downarrow}}{d \phi_{r}}} .
$$

The former (later) one is proportional to the real (imaginary) part of the linear combination that was mentioned. Both of them give sizeable effects. Although $A_{\mathrm{SL}}$ is proportional to the imaginary 

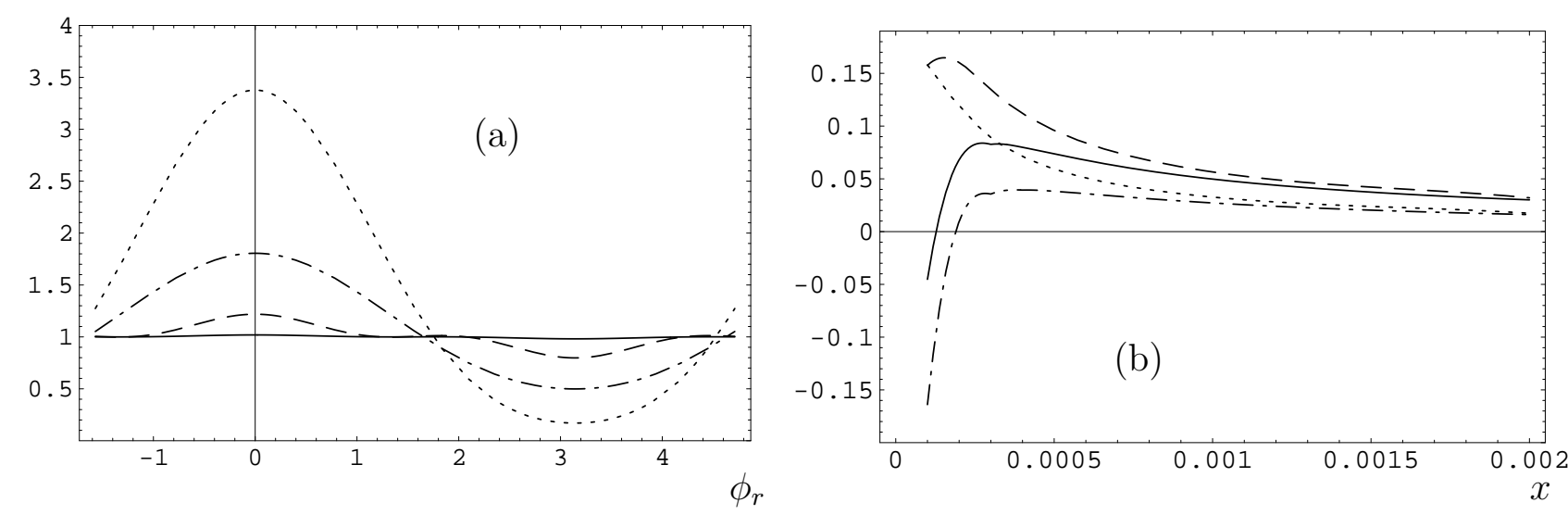

Figure 3: (a) Ratios of different approximations of the BH cross section to the exact one in dependence of the azimuthal angle $\phi_{r}$ for $\mathcal{Q}^{2}=4 \mathrm{GeV}^{2}$ and $x=10^{-4}$ for the Pade-type approximation for $\Delta^{2}=-0.1 \mathrm{GeV}^{2}$ (solid) and $\Delta^{2}=-0.5 \mathrm{GeV}^{2}$ (dashed) and for the leading approximation (27) (dash-dotted and dotted, respectively). (b) Unpolarized azimuthal angle asymmetry for $\Delta^{2}=-0.05(-0.25) \mathrm{GeV}^{2}$ versus $x$. Solid (dash-dotted) and dashed (dotted) lines show the result for the Pade and leading approximation, respectively, where $\kappa_{\text {sea }}$ is set to zero.

part which is growing as $x^{-1}$ this asymmetry is suppressed by a kinematical factor $y$ as compared to the charge asymmetry. Therefore, we find in the considered kinematics a two times larger value of the charge asymmetry as for the single spin one. This should be reversed for larger values of $y$

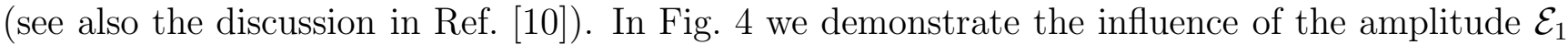
which is quite sizeable for larger values of $-\Delta^{2}$, where $-\Delta^{2} / \mathcal{Q}^{2}$ remains small.

To measure $\widetilde{\mathcal{H}}_{1}$ one have to consider the proton single spin asymmetry $\Delta_{\text {SLN }}=d \sigma_{\uparrow}-d \sigma_{\downarrow}$ given in Eq. (41):

$$
A_{\mathrm{SLN}}=\frac{\int_{0}^{\pi} d \phi_{r} \frac{\Delta_{\mathrm{SLN}} d \sigma}{d \phi_{r}}-\int_{\pi}^{2 \pi} d \phi_{r} \frac{\Delta_{\mathrm{SLN}} d \sigma}{d \phi_{r}}}{\int_{0}^{2 \pi} d \phi_{r} \frac{d \sigma_{\uparrow}+d \sigma_{\downarrow}}{d \phi_{r}}}
$$

and the charge asymmetry in double spin-flip experiments, i.e. $\Delta_{\mathrm{C}}^{\mathrm{DFL}} d \sigma=d^{-} \sigma_{\uparrow}^{\uparrow}-d^{+} \sigma_{\downarrow}^{\downarrow}-\Delta_{\mathrm{C}} d \sigma^{\mathrm{unp}}$, defined in Eq. (44)

$$
A_{\mathrm{C}}^{\mathrm{DFL}}=\frac{\int_{0}^{\pi} d \phi_{r} \frac{\Delta_{\mathrm{C}}^{\mathrm{DFL}} d \sigma}{d \phi_{r}}-\int_{\pi}^{2 \pi} d \phi_{r} \frac{\Delta_{\mathrm{C}}^{\mathrm{DFL}} d \sigma}{d \phi_{r}}}{\int_{0}^{2 \pi} d \phi_{r} \frac{d^{-} \sigma^{\mathrm{unp}}+d^{+} \sigma^{\mathrm{unp}}}{d \phi_{r}}} .
$$

Unfortunately, for the considered $\mathcal{Q}^{2}$ value we find for our model that this single spin asymmetry is compatible with zero. Although, we have an enhancement for small $y$ due do the factor $1 / y$ in comparison to the lepton single spin asymmetry, this enhancement cannot compensate the weaker rise of $\widetilde{\mathcal{H}}_{1}$ for small $x$. For larger value of $\mathcal{Q}$, i.e. also larger $x$, and $-\Delta^{2}$ and small $y$ we found a few percent effect. Note also that the ratio of proton to lepton single spin asymmetry gives us the 

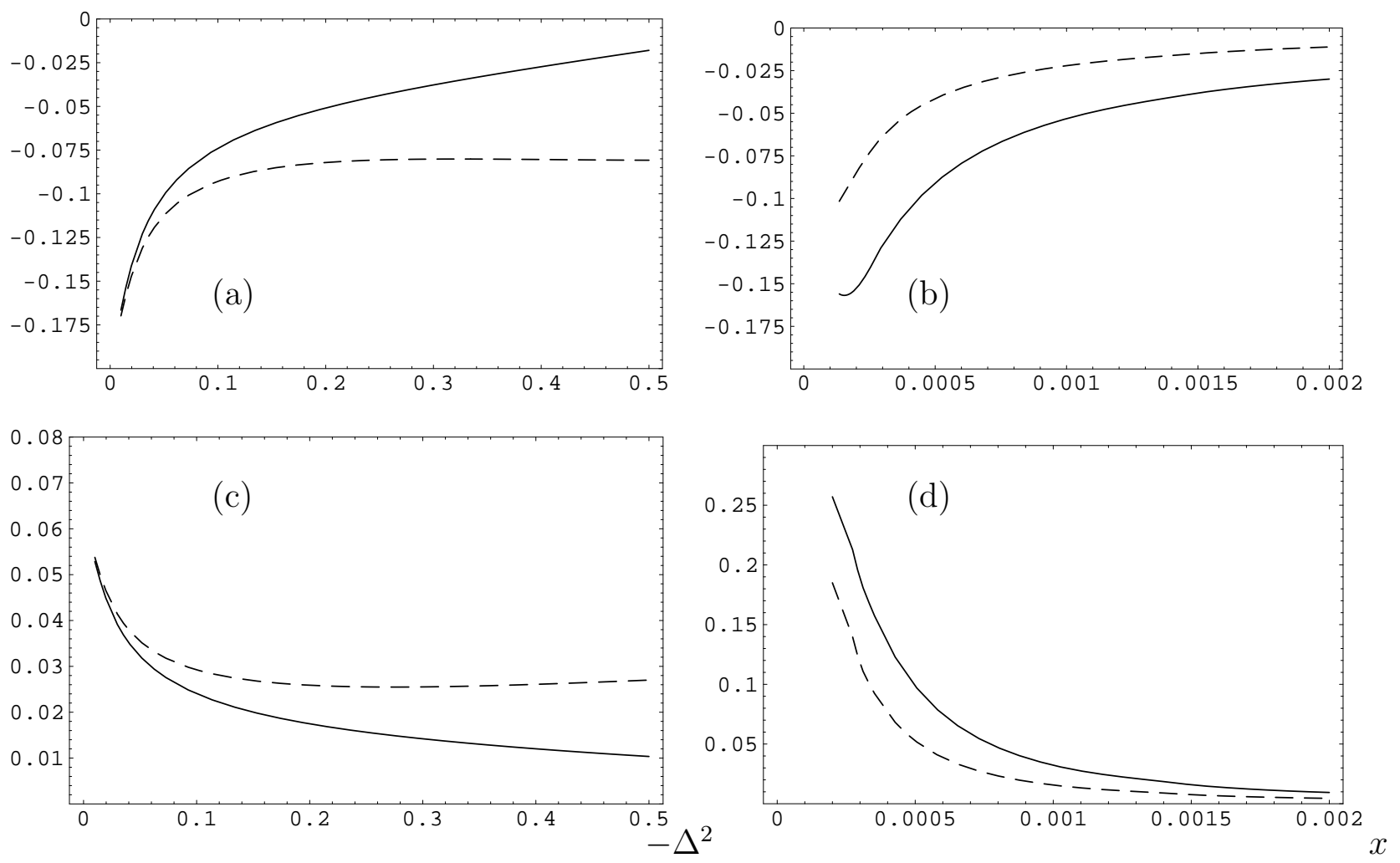

Figure 4: Unpolarized azimuthal charge asymmetry $A_{\mathrm{C}}$ for $\mathcal{Q}^{2}=4 \mathrm{GeV}^{2}$ and $x=5 \cdot 10^{-4}$ with $\kappa_{\text {sea }}=-2$ at LO for the complete expression (solid curve) and neglecting the $\mathcal{E}_{1}$ contribution (dashed curve) plotted in (a) versus $-\Delta^{2}$. The same asymmetry for $\Delta^{2}=-0.05 \mathrm{GeV}^{2}$ (solid curve) and $\Delta^{2}=-0.25 \mathrm{GeV}^{2}$ (dashed curve) at LO is shown in (b) for the region $1 \cdot 10^{-4} \leq x \leq 2 \cdot 10^{-3}$. In (c) and (d) the same is shown for the electron single spin asymmetry.

ratio $\left(\operatorname{Im} \widetilde{\mathcal{H}}_{1}\right) /\left(\operatorname{Im} \mathcal{H}_{1}\right)$ times $1 / y$, which is sizeable at not too small $x$. The azimuthal asymmetry in double spin flip experiments normalized to the unpolarized cross section is tiny. However, if we would compare it with the extracted double spin flip part, we would get quite sizeable effects which are of the order of $10 \%$ or even more. Furthermore, to explore $\mathcal{H}_{1}$ separately, one can make use of the discussion given in section 3.1. Especially, for small $y$ the BH cross section is suppressed by $y^{2}$ in comparison to the DVCS one and, thus, the former one drops out.

Let us note that the perturbative NLO corrections to the imaginary part for small $x$ are of order of $20 \%$ for each quark species as well as for the gluon contribution.

\subsection{HERMES kinematics.}

Now let us turn to the HERMES experiment with a $E=27.5 \mathrm{GeV}$ positron beam scattered by a hydrogen target and give predictions for the asymmetries which can be accesed there. To 

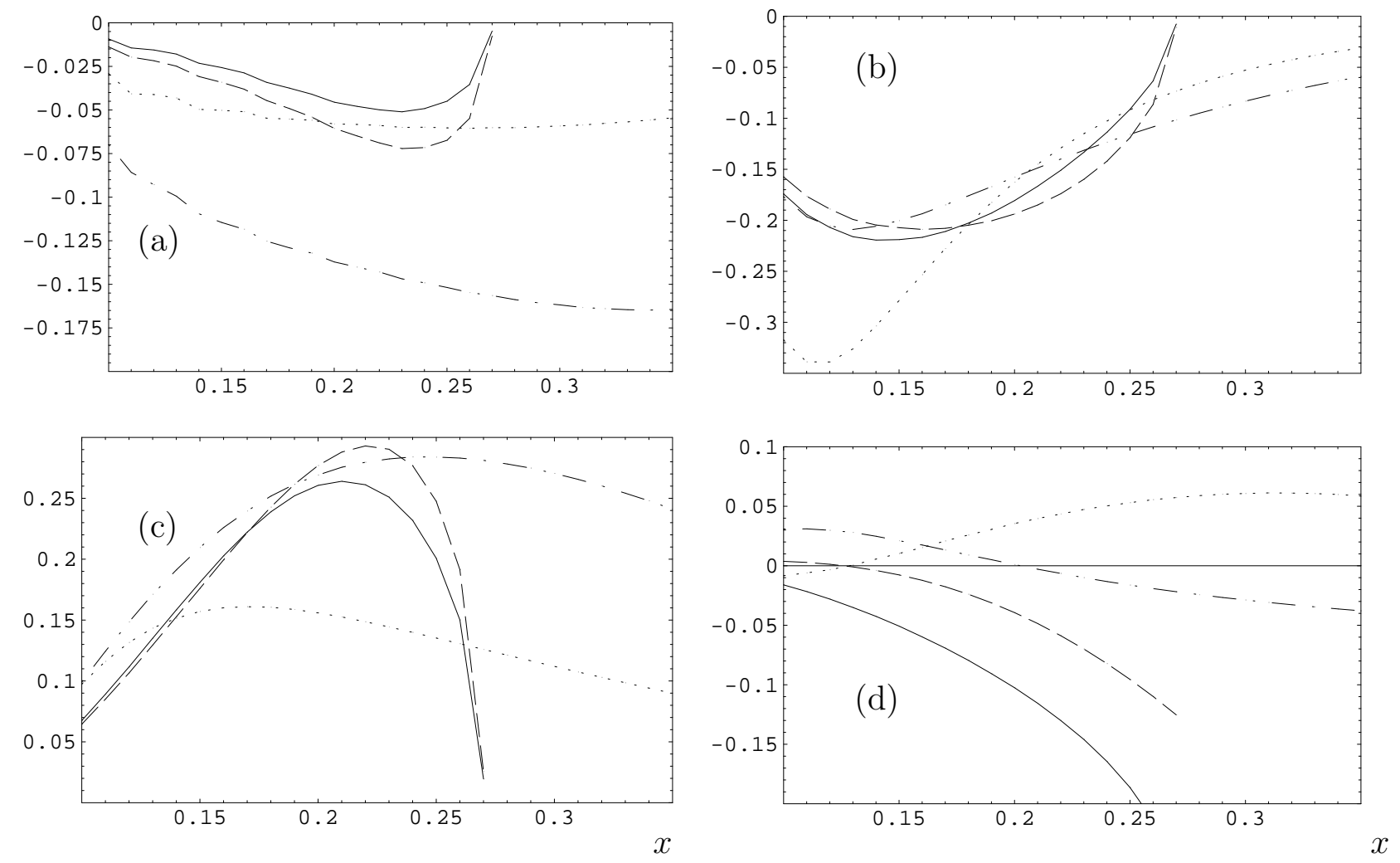

Figure 5: Perturbative leading order results for the charge asymmetry for an unpolarized beam (a), single spin asymmetries for a polarized positron beam (b) and an unpolarized target; as well as for an unpolarized lepton beam and a longitudinally (c) (transversally (d)) polarized proton target versus $x$, for $\mathcal{Q}^{2}=4 \mathrm{GeV}^{2}$. The predictions for the model specified in the text are shown as solid (dotted) curves for $\Delta^{2}=-0.1(0.5) \mathrm{GeV}^{2}$, respectively. The same model however with neglected spin-flip contributions are presented as dashed (dash-dotted) line for the same values of $\Delta^{2}$.

give numerical predictions we take the model for SPDs deduced from the double distribution model and forward (un-) polarized parton densities from Ref. (23]) [24] with $\kappa_{\text {sea }}=-2$. As a starting point we choose $\mathcal{Q}^{2}=4 \mathrm{GeV}^{2}, x$-range $0.1-0.4$, and the $t$-channel momentum transfer $-\Delta^{2}=0.1-0.5 \mathrm{GeV}^{2}$. Since the parton densities are defined at rather low momentum scale $\mathcal{Q}_{0}^{2}=$ $0.23 \mathrm{GeV}^{2}$ we evolve them up to the experimental scale using the formalism of 4 . We concentrate mostly on the cross sections proportional to the imaginary part of the DVCS amplitude. Since $\widetilde{E}_{1}$ is concentrated in the ER-BL region with zero at $|t|=\xi, \operatorname{Im} \widetilde{\mathcal{E}}_{1}$ vanishes at all order.

Similarly to the previous section we calculate the charge asymmetry for unpolarized experiments given in Eq. (59). As we see form the Fig. 5(a) it reaches the level of $5-15 \%$ for $-\Delta^{2}=0.5 \mathrm{GeV}^{2}$. The single lepton spin asymmetry (60) is much larger and can be as big as $20-30 \%$ [see Fig. 5(b)] which gives promises to measure it experimentally. Note that in both 
cases the cross sections are very sensitive to the helicity flip distribution $E$ which is responsible for the orbital momentum of partons in the proton.

In view of limited space let us consider finally only the spin asymmetry for a longitudinally and transversally polarized proton (42). In the former case azimuthal averaging of Eq. (41) is the same as in Eq. (60) and leads to a sizeable asymmetry of order 20\%. In the later case in order to extract the combination of SPDs multiplied by $\cos \left(\phi_{r} / 2\right)$ we define the following azimuthal asymmetry

$$
A_{\mathrm{STN}}=\frac{\int_{-\pi / 3}^{2 \pi / 3} d \phi_{r} \frac{\Delta_{\mathrm{STN}} d \sigma}{d \phi_{r}}-\int_{2 \pi / 3}^{5 \pi / 3} d \phi_{r} \frac{\Delta_{\mathrm{STN}} d \sigma}{d \phi_{r}}}{\int_{0}^{2 \pi} d \phi_{r} \frac{d \sigma_{\rightarrow}+d \sigma_{\leftarrow}}{d \phi_{r}}}
$$

The numerical estimate presented in Fig. 5 (d) demonstrates that it has a sizable effect which in contrast to the other symmetries does not vanish at the kinematical boundery $\Delta^{2}=\Delta_{\min }^{2}$.

\section{Conclusions.}

In this paper we have given theoretical predictions for diverse asymmetries which can be measured in exclusive leptoproduction experiments of a real photon. Our estimates are rather encouraging, since they demonstrate a possibility to separate the contributions coming from different leading twist-two DVCS amplitudes by means of polarized lepton beam, and longitudinally and transversally polarized targets. This is the first step on the way to constrain the form of the SPDs from experimental data. The models used for numerical estimates lead to large charge and single (lepton) spin asymmetries of order 20\%. However, since an asymmetry gives information on a linear combination of SPD one has to resort to other combinations of cross sections in order to disentangle a given distribution.

We have not discussed in the present paper the NLO correction to the DVCS amplitudes. However, as has been shown in Ref. 25] the latter could be very sizable and therefore change the LO predictions significantly in the valence quark region. Yet another important issue is the study of kinematical and dynamical higher twist corrections. Both of these problems deserve a detailed investigation and will be considered elsewhere.

We would like to thank M. Amarian, M. Diehl, and A.V. Radyushkin for constuctive correspondence. This work was supported by DFG and BMBF. D.M. thanks G. Sterman for the hospitality extended to him at the C.N. Yang Institute for Theoretical Physics while this work was finished. 


\section{References}

[1] D. Müller, D. Robaschik, B. Geyer, F.-M. Dittes, J. Hořejši, Fortschr. Phys. 42 (1994) 101.

[2] X. Ji, Phys. Rev. Lett. 78 (1997) 610; Phys. Rev. D 55 (1997) 7114; J. Phys. G 24 (1998) 1181.

[3] A.V. Radyushkin, Phys. Lett. B 380 (1996) 417; Phys. Rev. D 56 (1997) 5524.

[4] A.V. Belitsky, D. Müller, Nucl. Phys. B 537 (1999) 397;

A.V. Belitsky, D. Müller, L. Niedermeier, A. Schäfer, Nucl. Phys. B 546 (1999) 279;

A.V. Belitsky, D. Müller, A. Freund, Nucl. Phys. B 574 (2000) 347.

[5] P.R.B. Saull (ZEUS Collaboration), Talk at EPS99, Prompt Photon Production and Deeply Virtual Compton Scattering, Tampere, Finland (July 1999).

[6] P. Kroll, M. Schürmann, P.A.M. Guichon, Nucl. Phys. A 598 (1996) 435.

[7] M. Diehl, T. Gousset, B. Pire, J.P. Ralston, Phys. Lett. B 411 (1997) 193.

[8] L. Frankfurt, A. Freund, M. Strikman, Phys. Rev. D 58 (1998) 114001, (E) D 59 (1999) 119901.

[9] L. Frankfurt, A. Freund, M. Strikman, Phys. Lett. B 460 (1999) 417.

[10] A. Freund, M. Strikman, Phys. Rev. D 60 (1999) 071501.

[11] P. Hoodbhoy, X. Ji, Phys. Rev. D 58 (1998) 054006.

[12] A.V. Belitsky, D. Müller, Phys. Lett. B 486 (2000) 369.

[13] L.B. Okun, Leptons and quarks, North-Holland, (Amsterdam, 1982).

[14] C. Itzykson, J. Zuber, Quantum Field Theory, McGraw-Hill, (New York, 1980).

[15] I. Anikin, B. Pire, O. Teryaev, Phys. Rev. D 62 (2000) 071501.

[16] A.V. Radyushkin, Phys. Rev. D 59 (1999) 014030.

[17] A.V. Belitsky, D. Müller, Nucl. Phys. B (Proc. Suppl.) 79 (1999) 573, hep-ph/9905263.

[18] L. Mankiewicz, G. Piller, A.V. Radyushkin, Eur. Phys. J. C 10 (1999) 307.

[19] L.L. Frankfurt, P.V. Pobylitsa, M.V. Polyakov, M. Strikman, Phys. Rev. D 60 (1999) 0140010. 
[20] A.D. Martin, R.G. Roberts, W.J. Stirling, Phys. Lett. B 354 (1995) 155.

[21] T. Gehrmann, W.J. Stirling, Phys. Rev. D 53 (1996) 6100.

[22] I.I. Balitsky, E. Kuchina, Phys. Rev. D 62 (2000) 074004.

[23] M. Glück, E. Reya, A. Vogt, Z. Phys. C 67 (1995) 433.

[24] M. Glück, E. Reya, M. Stratmann, W. Vogelsang, Phys. Rev. D 53 (1996) 4775.

[25] A.V. Belitsky, D. Müller, L. Niedermeier, A. Schäfer, Phys. Lett. B 474 (2000) 163. 